\title{
Remote Sensing from Geostationary Orbit: GEO TROPSAT, A New Concept for Atmospheric Remote Sensing
}

\author{
Alan D. Little ${ }^{\mathrm{a}}$, Doreen O. Neil ${ }^{\mathrm{a}}$, Glen W. Sachse ${ }^{\mathrm{a}}$, Jack Fishman ${ }^{\mathrm{a}}$, Arlin J. Krueger ${ }^{\mathrm{b}}$ \\ ${ }^{a}$ NASA Langley Research Center, M/S 468, Hampton VA 23681-0001 \\ ${ }^{b}$ NASA Goddard Space Flight Center
}

\begin{abstract}
The Geostationary Tropospheric Pollution Satellite (GEO TROPSAT) mission is a new approach to measuring the critical constituents of tropospheric ozone chemistry: ozone, carbon monoxide, nitrogen dioxide, and aerosols. The GEO TROPSAT mission comprises a constellation of three instruments flying as secondary payloads on geostationary communications satellites around the world. This proposed approach can significantly reduce the cost of getting a science payload to geostationary orbit and also generates revenue for the satellite owners. The geostationary vantage point enables simultaneous high temporal and spatial resolution measurement of tropospheric trace gases, leading to greatly improved atmospheric ozone chemistry knowledge. The science data processing, conducted as a research (not operational) activity, will provide atmospheric trace gas data many times per day over the same region at better than $25 \mathrm{~km}$ ground footprint. The high temporal resolution identifies short time scale processes, diurnal variations, seasonal trends, and interannual variation.
\end{abstract}

Keywords: space mission design, atmospheric processes, geostationary, focal plane array applications, tropospheric pollution, secondary payload, communications satellite, GEO TROPSAT, Geo Express Pathfinder

\subsection{INTRODUCTION}

Space based atmospheric remote sensing has traditionally examined the upper atmosphere. Recent changes in NASA's science strategies have led to increased focus on measurements in the more highly variable troposphere. This paper will propose a new concept to examine tropospheric ozone chemistry. NASA currently utilizes low earth orbit for its Earth observing missions. We discuss a new opportunity to deploy science instruments in geostationary orbit. Geostationary orbital positions have great commercial value, and are too expensive for many science missions. However, access to these geostationary positions can be obtained for science sensors flying as secondary payloads on commercial geostationary satellites.

This paper is organized into five major sections. Section 2 presents the new mission concept for atmospheric remote sensing. This section introduces the advantages of geostationary orbit observations, and characteristics of secondary payloads. Section 3 discusses the proposed GEO TROPSAT science mission, why the mission is scientifically important, and the rationale for the mission. The measurement parameters, selection, methods and goals will be presented. Section 4 discusses the mission characteristics that are a function of the unique geostationary vantage point. This section will cover the viewing geometry, and radiance calculations. Section 5 presents the conceptual GEO TROPSAT instrument, which consists of two nadir viewing filter cameras: GEO-CO and GEO-O3.

\subsection{MISSION CONCEPT}

Chemistry of the troposphere is highly complex. It comprises "point" and distributed sources (of natural and anthropogenic origin); complicated transport processes (both lateral and vertical); and photochemistry driven by ultraviolet flux, temperature, atmospheric composition and dynamics, and other variables. These sources, transport, and photochemical processes vary significantly in space and time. The best understanding of these processes requires observations that simultaneously possess high spatial and temporal resolution. Efforts have been made to provide high spatial resolution (10 $\mathrm{x}$ $10 \mathrm{~km}^{2}$ ) from low earth orbit (LEO), but the available temporal resolution from LEO (1 day or more) is inadequate to understand crucial processes which have diurnal time scales. In addition, the "global" picture obtained from LEO (even for instruments that revisit the same spot on Earth every 24 hours) is a convolution of the true, but rapidly changing, tropospheric state. The LEO perspective further complicates the interpretation of the already complex troposphere. 
Geostationary Earth orbit (GEO) provides the ability to "stare" at a portion of the Earth continuously. Early observations from GEO were limited by single element detector systems that required scanning to view the entire scene. Consequently, such observations could not exploit the unique property of GEO: continuous, simultaneous observations over large expanses of the Earth. Recent years have brought advances in 2-D imaging arrays for scientific, defense and video use. At GEO, the combination of continuous temporal access and large 2-D imaging arrays provides the simultaneous high spatial and high temporal resolution required to advance tropospheric studies with an accurate visualization of sources, sinks, and transport processes.

NASA has developed access to LEO for science missions, but access to GEO has been an obstacle for science measurements. The geostationary operational meteorological satellites perform high value, tightly focused science measurements in an environment that demands high reliability on a schedule not tailored for research. Research science investigations must find another method to reach GEO. More recently, the enormous growth in telecommunications (direct broadcast TV, cellular telephones, and video on demand) has required telecommunications businesses to increase launch frequency and performance capability of their systems to maximize the use of limited geostationary orbit locations around the world. The resource demands of selected Earth remote sensing instruments have become trivial in comparison to the capabilities of these spacecraft. Consequently, a new method of access to geostationary orbit is now possible: science sensors as secondary payloads on commercial communications spacecraft.

A striking characteristic of the GEO TROPSAT mission concept is its access to geostationary orbit as secondary payloads on commercial communications satellites, rather than requiring dedicated satellites. The motivation for this departure from standard practice derives from the present fiscal constraints on science investigations, and from the effective infrastructure assembled by the commercial industry which results in more than 25 successful new satellites in GEO annually. Global coverage (multiple instruments) becomes fiscally possible as a secondary payload, and the international market provides flight opportunities around the globe. The commercial satellite provides nadir view, electrical power, thermal environment, attitude, command uplink, and data downlink as on-orbit services for the secondary payload. Leasing these on-orbit services appears to be financially attractive to both NASA and the commercial satellite owners. NASA Mission To Planet Earth (MTPE) is currently funding a pathfinder project at NASA Langley Research Center to develop the business practices required to enable secondary payloads on commercial geostationary satellites. This pathfinding project, Geo Express Pathfinder (GXP), will measure total column ozone and expects to be launched in 1999.

Secondary payload status also imposes significant constraints on the science instrument. Physical size, mass and schedule are the gatekeepers to successful secondary payload opportunities. Because GEO TROPSAT operates in the beginning-oflife margins of the spacecraft, its on-orbit life is constrained by the design and performance of the satellite. The present generation of commercial communications spacecraft appears capable of supporting at least two years of on-orbit services for GEO TROPSAT without affecting the primary commercial mission of the satellite. On time delivery of the secondary payload is mandatory, and the commercial satellite will launch without the secondary payload in order to maintain schedule. Interaction with the commercial satellite operator is required to command the secondary payload, driving the science payload to be highly autonomous. Occasionally, the secondary payload may endure a loss of power because of operational needs of the commercial payload. Even with these constraints, the enhanced science capability afforded by secondary payloads at GEO propels the study of tropospheric processes into a new era.

\subsection{GEO TROPSAT SCIENCE}

GEO TROPSAT provides concurrent measurements of ozone, carbon monoxide, aerosols, and nitrogen dioxide, and analysis of the tropospheric contribution. Together with atmospheric state information (temperature, pressure, water vapor), these constituents indicate the radiative balance, dynamics, and chemical state of the atmosphere. Although the state information from operational meteorological satellites has become routinely available, little advancement has been made in satellite measurements of other tropospheric constituents. This paucity of spaceborne tropospheric measurements contrasts sharply with the highly successful long term monitoring of many stratospheric species from the Upper Atmospheric Research Satellite (UARS), Total Ozone Mapping Spectrometer (TOMS), and the Stratospheric Aerosol and Gas Experiment (SAGE) for example. The troposphere presents a more difficult research environment because of its high variability in space and time, the presence of clouds (which significantly affects the choice of measurement technique and complicates interpretation of the data), and the high degree of spectral line overlap in the real troposphere. Consequently only a few meaningful

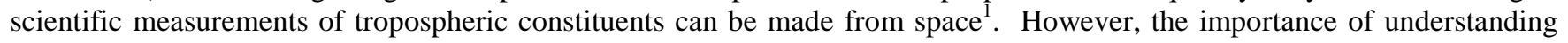
the delicate part of the atmosphere in which we live remains compelling in the face of these challenges. 
Ozone is the primary chemical precursor of tropospheric $\mathrm{OH}$, and $\mathrm{CO}$ is the dominant sink of $\mathrm{OH}$ in the troposphere. Together, these two species dominate the oxidizing (cleansing) capacity of the atmosphere. Pollution sources provide high levels of $\mathrm{CO}$, making it an excellent tracer of human impact on the atmosphere, and enabling visualization of transport in the troposphere. Although ozone in the stratosphere provides life-sustaining protection from ultraviolet radiation, ozone in the biosphere is toxic to living things. In the upper troposphere, ozone is a significant greenhouse gas, altering the radiative balance of the planet and affecting long-term climate change. Nitrogen dioxide production is linked to photo-oxidation processes involving $\mathrm{OH}$. Aerosols provide sites for chemical reactions, and alter the radiative behavior of the atmosphere. The critical science need is to understand the processes that determine the spatial distributions and temporal changes of tropospheric ozone chemistry. Because of the short lifetimes and highly variable concentrations of these constituents, GEO provides the unique vantage to map distributions, determine diurnal variability, and observe transport. The primary science goal of GEO TROPSAT is the simultaneous observation of urban, remote continental, and ocean areas to study emission and transport on local and regional scales.

Constituent retrievals are based on significant LEO heritage. TOMS algorithms ${ }^{2}$ and wavelengths are used to identify total ozone, stratospheric ozone, and aerosols, and to estimate cloud top heights. GOME algorithms ${ }^{3}$ provide $\mathrm{NO}_{2}$. $\mathrm{CO}$ retrievals ${ }^{4}$ in the near infrared utilize the technique of Rodgers. GEO TROPSAT's measurements are highly complementary with those of NASA's Earth Observing System, which will provide more detailed vertical resolution of the distribution of these constituents. In addition, the operational meteorological satellites provide vital ancillary data for GEO TROPSAT's science studies.

Employing large format focal plane arrays, GEO TROPSAT is able to image the viewed portion of the Earth at $12-25 \mathrm{~km}$ ground footprint without scanning. Building up a series of still images of the same scene, taken multiple times per hour, GEO TROPSAT's measurements will reveal the processes of atmospheric ozone chemistry for detailed quantitative study. A constellation of three satellites, distributed around Earth, provides continuous global coverage.

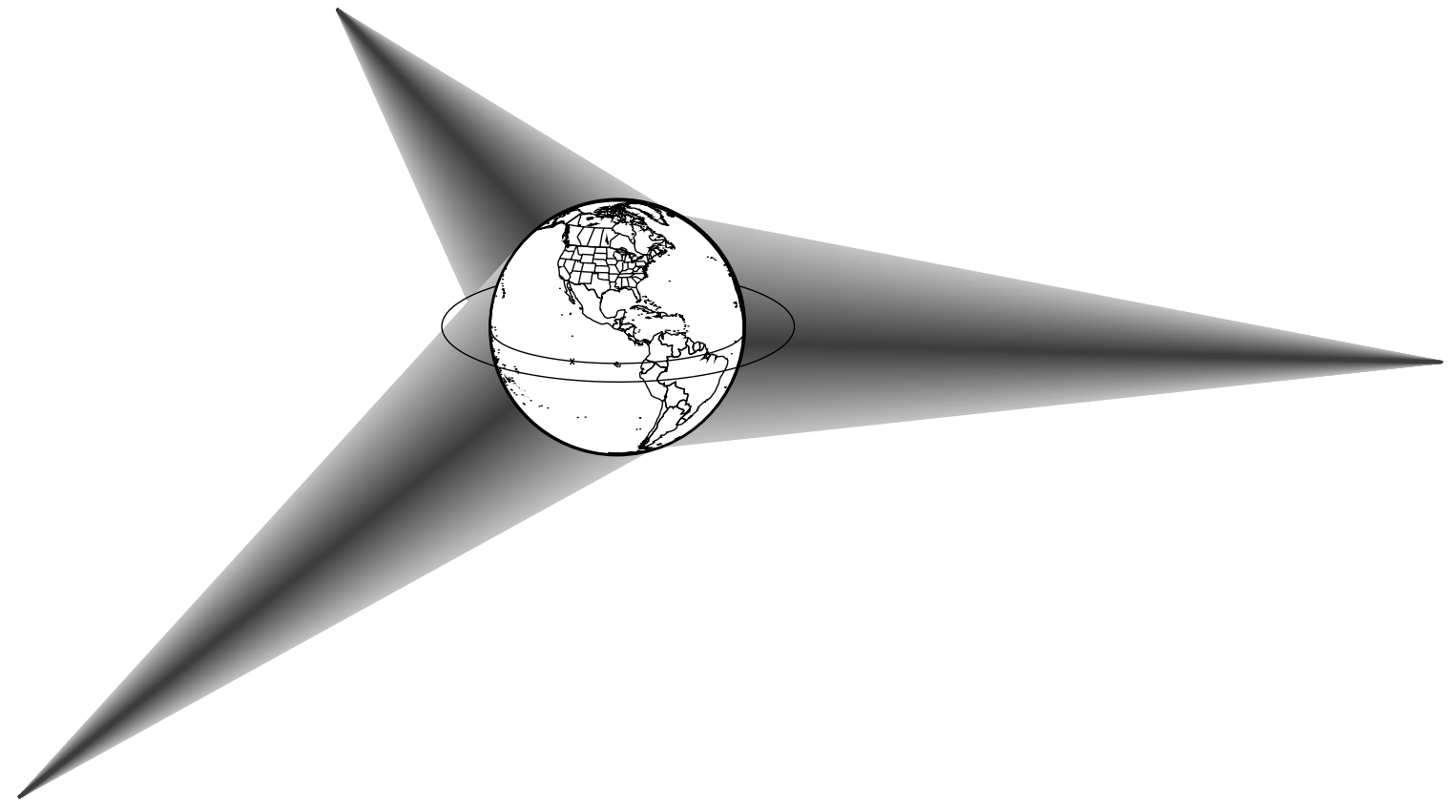

Figure 1 GEO TROPSAT constellation. Global coverage is provided by instruments located at $110^{\circ} \mathrm{W}, 10^{\circ} \mathrm{E}$, and $130^{\circ} \mathrm{E}$.

GEO TROPSAT is committed to using tropospheric data for public benefit. There are three dimensions to this effort: increase public understanding of the fundamental processes leading to the formation and accumulation of ozone in the lower atmosphere; enhance science education of the next generation of scientists and engineers by involving students in data collection and interpretation; and provide immediate visualization of air quality products via The Weather Channel ${ }^{\mathrm{TM}}$ for use in the public's daily activities.

As an example of the first dimension of GEO TROPSAT's public outreach (understanding), GEO TROPSAT can provide data for regional planning teams to use in designing comprehensive plans to meet national air quality standards for ozone and 
particulates that are more effective and less costly to the public. GEO TROPSAT's public education dimension is addressed by planned partnerships with NASA's Classroom of the Future (www.cotf.edu) and with the Global Learning and Observations to Benefit the Environment (GLOBE) Project (www.globe.gov). Visualizations of air quality measurements can be made familiar to the public when presented in a format consistent with weather reports on television by The Weather Channel ${ }^{\mathrm{TM}}$.

\subsection{MISSION GEOMETRY}

The GEO TROPSAT mission proposes to deploy a constellation of instruments to geostationary orbit. The constellation will consist of three equally spaced instruments, nominally located at $110^{\circ} \mathrm{W}, 10^{\circ} \mathrm{E}$, and $130^{\circ} \mathrm{E}$, to provide global coverage as shown in Figure 1. Geostationary altitude $(35,800 \mathrm{~km})$ provides a very different viewing geometry than LEO. GEO TROPSAT's two nadir viewing sensors, GEO-CO and GEO-O3, are designed to take advantage of the geostationary geometry. The field of view is sized to observe the entire Earth disk as well as a portion of deep space around the Earth as shown in Figure 2. The GEO-CO and GEO-O3 sensors are designed with matched, 20 degree (0.349 radians), fields of view.

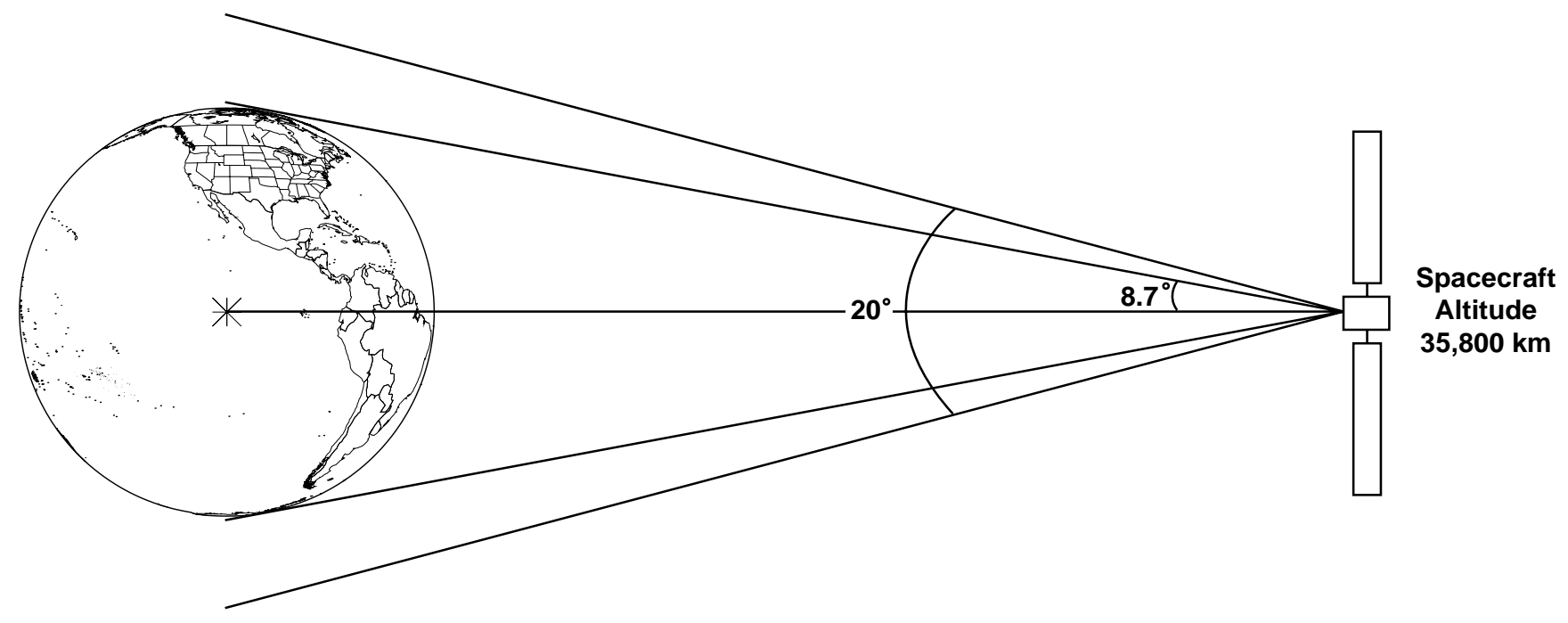

Figure 2 GEO TROPSAT Field of View.

The two sensors also have identical instantaneous fields of view of 0.0195 degrees (341 $\mu$ radians). The matched fields of view simplify the tropospheric chemistry analysis utilizing both the ozone and carbon monoxide measurements. The oversized field of view accommodates spacecraft attitude variations and allows for the viewing of the Earth limb and stars. GEO TROPSAT will use a combination of several techniques, star tracking, limb sensing, and feature recognition, to
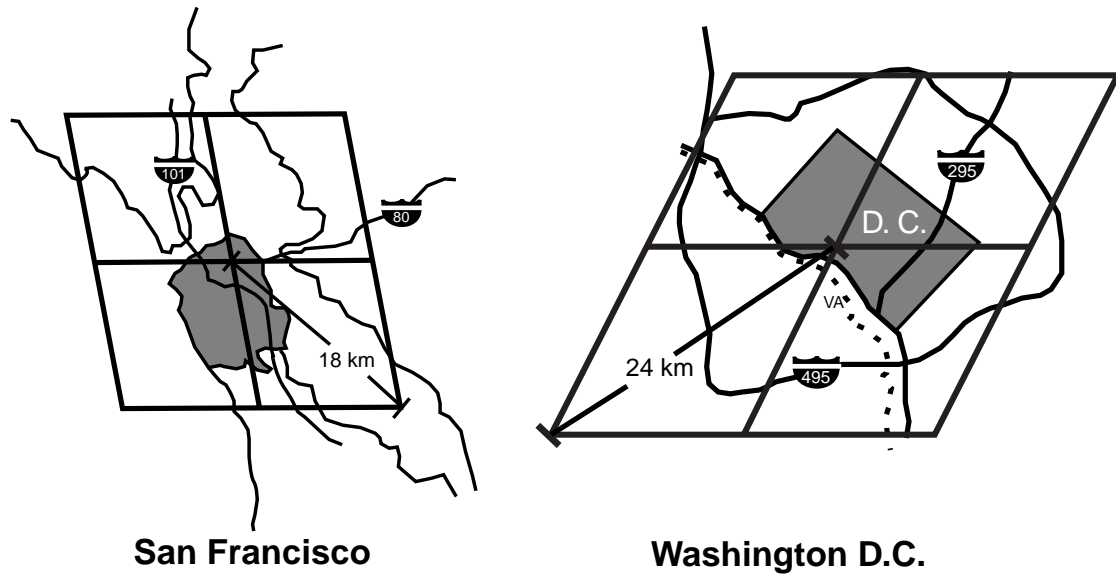

Washington D.C. geolocate the image. The geolocation information will be extracted from the science data images. The geostationary altitude and the sensor's instantaneous field of view define the size and shape of the observed ground footprint. The nadir pixel is $12 \mathrm{~km}$ square. Pixels along the equator are rectangular with the rectangle growing in length as the distance from the nadir point increases. Similarly, pixels along the spacecraft meridian are also rectangular with increasing latitude. Pixels that do not lie on the equator or the spacecraft meridian have various parallelogram shapes with the diagonal dimension increasing as the radial distance from the nadir point increases. Figure 3 illustrates the shapes of the pixels

Figure 3 San Francisco and Washington DC pixel shapes and dimensions. 
and their dimensions over the San Francisco and Washington DC areas. Figure 4 displays a contour map of the footprint radial dimension on a global scale. The inner contours are $15 \mathrm{~km}$ and increase to the outer contours of $75 \mathrm{~km}$ as labeled. This geometry provides for a maximum footprint dimensions of $25 \mathrm{~km}$ or less over the majority of the inhabited areas of the planet.

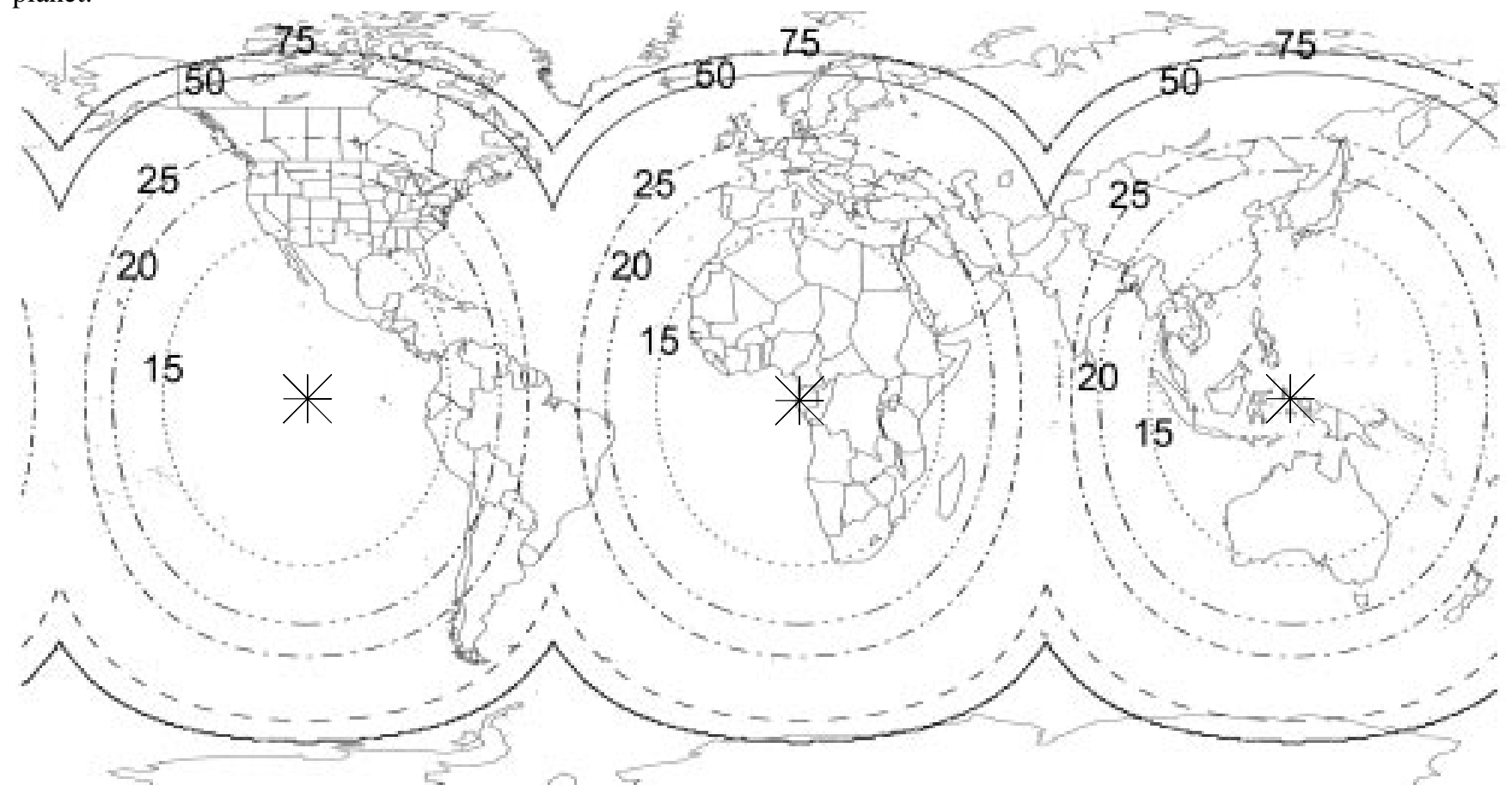

Figure 4 GEO TROPSAT constellation radial footprint dimension contour map. The footprint increases with the radial distance from the nadir point (denoted by the *). (Note: All contour levels are in $\mathbf{k m}$.)

The GEO TROPSAT sensors utilize reflected sunlight to measure the trace gases. The relationship of the sun to the spacecraft in geostationary orbit differs from low earth orbit spacecraft. Geostationary measurements are inherently non-sunsynchronous. GEO TROPSAT's measurements are only possible when the solar zenith angle is larger than $\approx 80$ degrees,

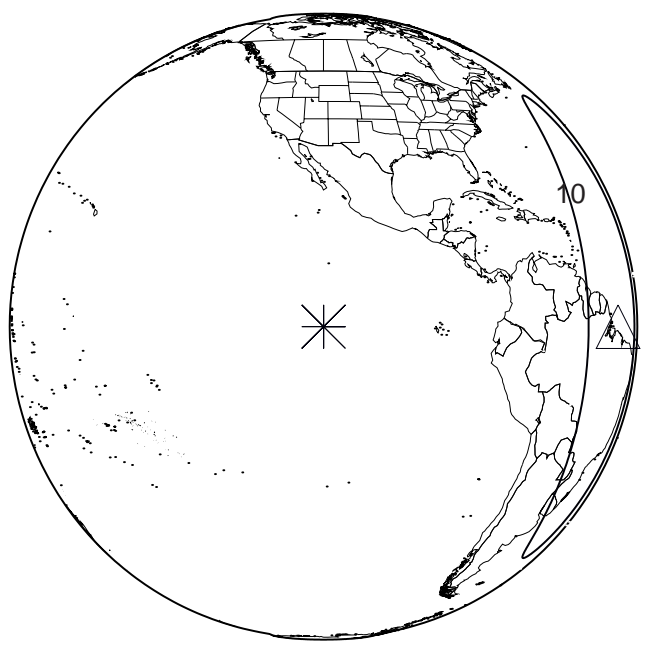

a. 3:00 AM

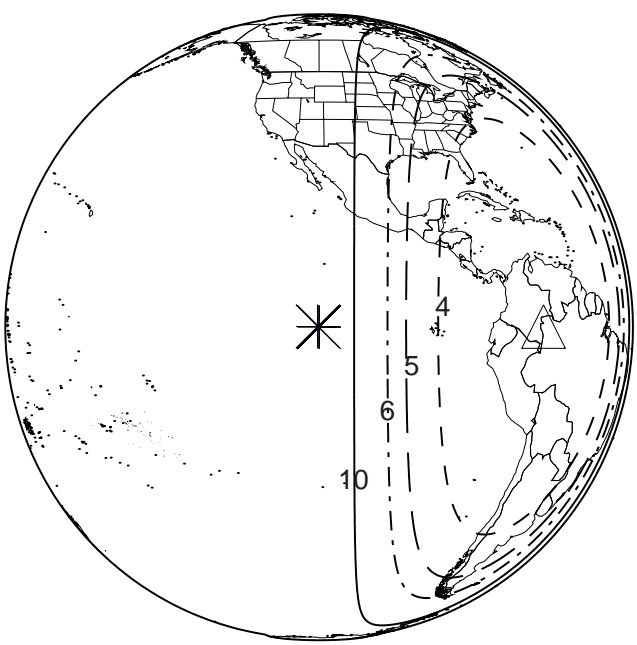

b. 6:00 AM

Figure 5 a-b Airmass contour maps illustrating the illuminated portion of the Earth at 3:00 AM and 6:00 AM satellite time. The nadir point is marked with the asterisk and the minimum airmass point is marked with the triangle. (Note: all contours are in units of airmass.) 


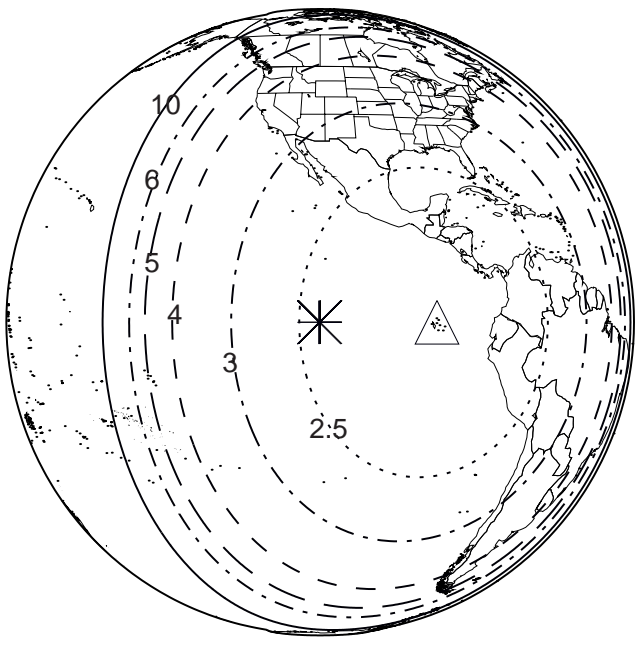

c. 9:00 AM

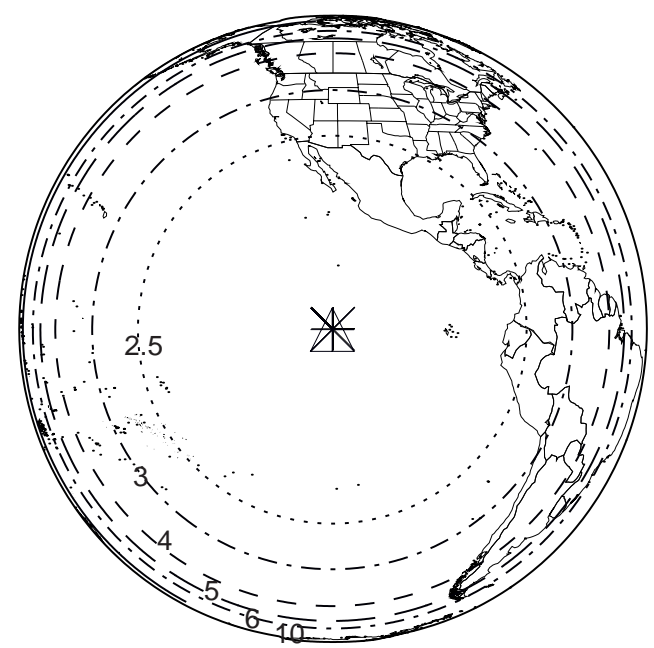

d. 12:00 PM

Figure 5 c-d Airmass contour maps illustrating the illuminated portion of the Earth at 9:00 AM and 12:00 PM satellite time. The nadir point is marked with the asterisk and the minimum airmass point is marked with the triangle. (Note: all contours are in units of airmass.)

corresponding to approximately dawn and sunset at the target location. The sun illuminates various portions of the Earth during the course of the day. Figures 5 a-d show the illuminated portions of the Earth as well as the corresponding airmass as a function of satellite time of day at the equinoxes. The airmass unit is defined as the path length through the atmosphere for a nadir-viewing instrument. The first figure (Figure 5a) shows the sun just rising over the eastern portion of the viewed area at 3:00 AM satellite time. The second figure (Figure 5b) shows the increase in the illuminated area at 6:00 AM satellite time, the third figure (Figure 5c) shows that the Earth is almost completely illuminated at 9:00 AM satellite time. The last figure (Figure 5d) shows the illumination at satellite noon. The illuminated areas decrease as the day continues on. There are also seasonal variations in the illuminated portions, where the illuminated portion shifts north or south, which are not shown here.

Radiance calculations, using MODTRAN 3.1 (1976 US Standard Atmosphere), have been made for each of the channels. The radiance data has been used to generate an initial engineering model of the instrument performance and to bound the expected radiance. The radiance calculations will be verified using more precise line-by-line radiative transfer calculations. Minimum radiance is based on low illumination (near dawn or dusk), reflected off the ocean, in a clear 1976 US Standard Atmosphere (23-km visibility). The maximum radiance case is based on high illumination (local noon), off a high reflectivity surface (cumulus clouds). The calculated minimum and maximum radiances at the top of the atmosphere are shown for selected channels in Table 1.

\begin{tabular}{|c|c|c|c|c|c|}
\hline \multicolumn{6}{|c|}{ Selected Radiance and Photon Flux Calculations } \\
\hline Wavelength (nm) & $\begin{array}{l}\text { Channel Width } \\
\text { (nm) }\end{array}$ & Condition $^{\mathrm{a}}$ & $\begin{array}{l}\text { Solar Zenith } \\
\text { Angle (deg) }\end{array}$ & $\begin{array}{l}\text { TOA Radiance } \\
\left(\mathrm{W} /\left(\mathrm{cm}^{2} *_{\mathrm{sr}}\right)\right)\end{array}$ & $\begin{array}{c}\text { Photon Flux }{ }^{\mathrm{b}} \\
\text { (Photons)/(sec*ifov) }\end{array}$ \\
\hline \multirow{3}{*}{317} & \multirow[t]{3}{*}{ (2) } & Max. Radiance & 0 & $1.21 \mathrm{E}-05$ & $5.49 \mathrm{E}+06$ \\
\hline & & & 70 & 1.35E-06 & $6.12 \mathrm{E}+05$ \\
\hline & & Min. Radiance & 85 & 1.34E-07 & $6.06 \mathrm{E}+04$ \\
\hline \multirow{3}{*}{322} & \multirow{3}{*}{1} & Max. Radiance & 0 & $1.24 \mathrm{E}-05$ & $5.62 \mathrm{E}+06$ \\
\hline & & & 70 & 1.65E-06 & $7.47 \mathrm{E}+05$ \\
\hline & & Min. Radiance & 85 & $2.25 E-07$ & $1.02 \mathrm{E}+05$ \\
\hline \multirow{3}{*}{329} & \multirow{3}{*}{1} & Max. Radiance & 0 & $2.32 E-05$ & $1.07 \mathrm{E}+07$ \\
\hline & & & 70 & $3.98 \mathrm{E}-06$ & $1.83 \mathrm{E}+06$ \\
\hline & & Min. Radiance & 85 & 9.95E-07 & $4.56 \mathrm{E}+05$ \\
\hline
\end{tabular}




\begin{tabular}{|c|c|c|c|c|c|}
\hline \multicolumn{6}{|c|}{ Selected Radiance and Photon Flux Calculations (continued) } \\
\hline Wavelength (nm) & $\begin{array}{c}\text { Channel Width } \\
(\mathrm{nm})\end{array}$ & Condition $^{\mathrm{a}}$ & $\begin{array}{l}\text { Solar Zenith } \\
\text { Angle (deg) }\end{array}$ & $\begin{array}{l}\text { TOA Radiance } \\
\left(\mathrm{W} /\left(\mathrm{cm}^{2} * \mathrm{sr}\right)\right)\end{array}$ & $\begin{array}{c}\text { Photon Flux }{ }^{\mathrm{b}} \\
\text { (Photons)/(sec*ifov) }\end{array}$ \\
\hline \multirow{3}{*}{360} & \multirow{3}{*}{1} & Max. Radiance & 0 & $3.12 \mathrm{E}-05$ & $1.43 \mathrm{E}+07$ \\
\hline & & & 70 & $4.23 \mathrm{E}-06$ & $1.94 \mathrm{E}+06$ \\
\hline & & Min. Radiance & 85 & $1.38 \mathrm{E}-06$ & $6.34 \mathrm{E}+05$ \\
\hline \multirow{3}{*}{2345} & \multirow{3}{*}{70} & Max. Radiance & 0 & 2.69E-05 & $8.99 \mathrm{E}+07$ \\
\hline & & & 70 & 5.35E-07 & $1.78 \mathrm{E}+06$ \\
\hline & & Min. Radiance & 85 & 1.76E-07 & $5.87 \mathrm{E}+05$ \\
\hline
\end{tabular}

Table 1 Selected GEO TROPSAT radiance and photon flux calculations.

a. The maximum radiance condition corresponds to a nadir view angle and cumulus clouds. The minimum radiance condition corresponds to an ocean view.

b. F/2 optical design, $12 \mu \mathrm{m}$ pixels for the ultraviolet-visible channels, and $18.5 \mu \mathrm{m}$ pixels for the near IR channel.

\subsection{INSTRUMENT CONCEPT}

The GEO TROPSAT instrument consists of two co-aligned, relatively simple, nadir viewing sensors: GEO-CO (primary measurement is total column carbon monoxide) and GEO-O3 (primary measurement is total column ozone). The GEO-O3 and GEO-CO sensors are passive filter cameras that rely upon reflected solar radiation as shown in Figure 6 . GEO TROPSAT will acquire images during the daylit portions of the day: up to 16 hours a day. Each sensor has its own optical and detector system and shares a common electronics system. The combined instrument mass is less than $40 \mathrm{kgs}$ and the instrument draws less than 250 watts of power. A functional block diagram of the system is shown in Figure 7 . The block diagram of the instrument shows the flow of information throughout the instrument, which is orchestrated by the command

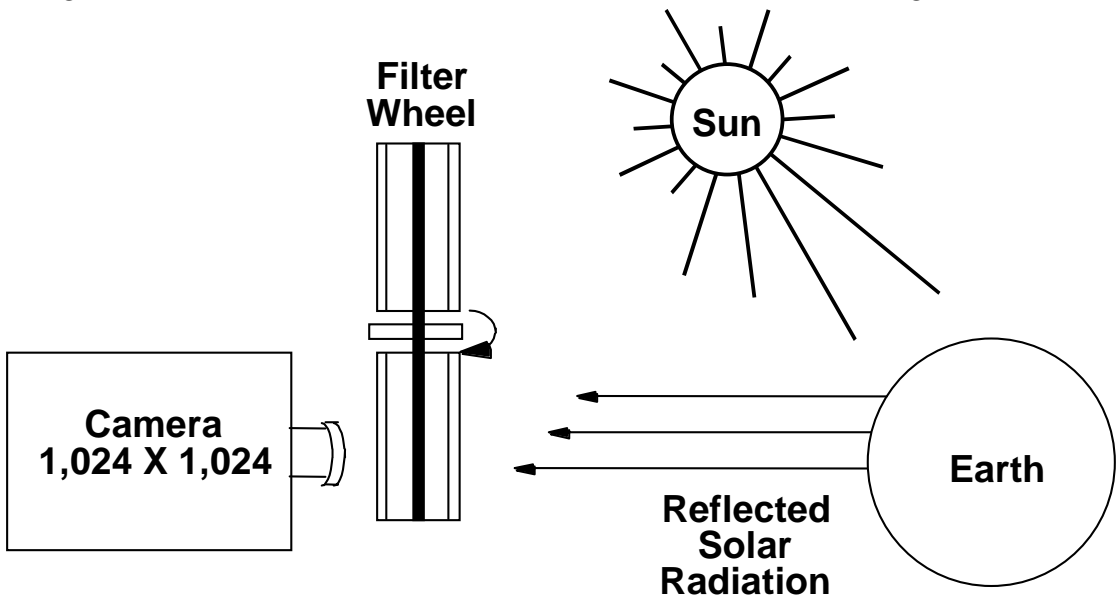

Figure 6 Representation of GEO TROPSAT filter cameras. and control system. The spacecraft provides commanding (through the 1553 interface), power, nadir field of view, attitude information, and the thermal environment. Commands are uplinked to the instrument through the spacecraft operator's telemetry system and relayed to the instrument over the spacecraft's 1553 data bus. The two sensors operate sequentially utilizing the same command and control electronics, digital signal processor, mass storage, and telemetry system. The integration time varies for each channel in order to maximize the system performance. The integration times are set to utilize the full dynamic range of the instrument and maximize the signal collected (i.e. operate with the detector wells nearly full).

\section{GEO-O3 Sensor}

The GEO-O3 sensor has 14 active channels in the spectral regions shown in Table 2. The ozone filter camera is based upon a modified Schwartzchild design optical system with the interference filter wheel located close to the focal plane. The filter wheel is used in a step and stare mode, in which a filter is positioned in front of the CCD array (step), the radiance is detected and read out (stare). The filter wheel remains positioned and images are taken until the desired signal to noise has been obtained. Then the filter wheel is moved to the next channel. The UV-visible camera uses a thermoelectrically cooled, back thinned, UV enhanced 1024x1024 Silicon CCD array. The CCD array is cooled to $220 \mathrm{~K}$ to minimize the dark current. 


\begin{tabular}{|c|c|l||}
\hline \multicolumn{3}{|c|}{ GEO-O3 Sensor Spectral Bands } \\
\hline Spectral Range (nm) & Number of Channels & \multicolumn{1}{c|}{ Function } \\
\hline $310-360$ & 5 & Total column ozone, sulfur dioxide, aerosols, albedo \\
\hline $295-305$ & 1 & Upper level ozone, volcanic cloud height \\
\hline $390-410$ & 2 & Cloud top height \\
\hline $435-450$ & 2 & Total column nitrogen dioxide \\
\hline $500-600$ & 3 & Chappuis band total column ozone \\
\hline $740-760$ & 1 & Sulfate aerosols \\
\hline
\end{tabular}

Table 2 GEO-O3 sensor spectral regions

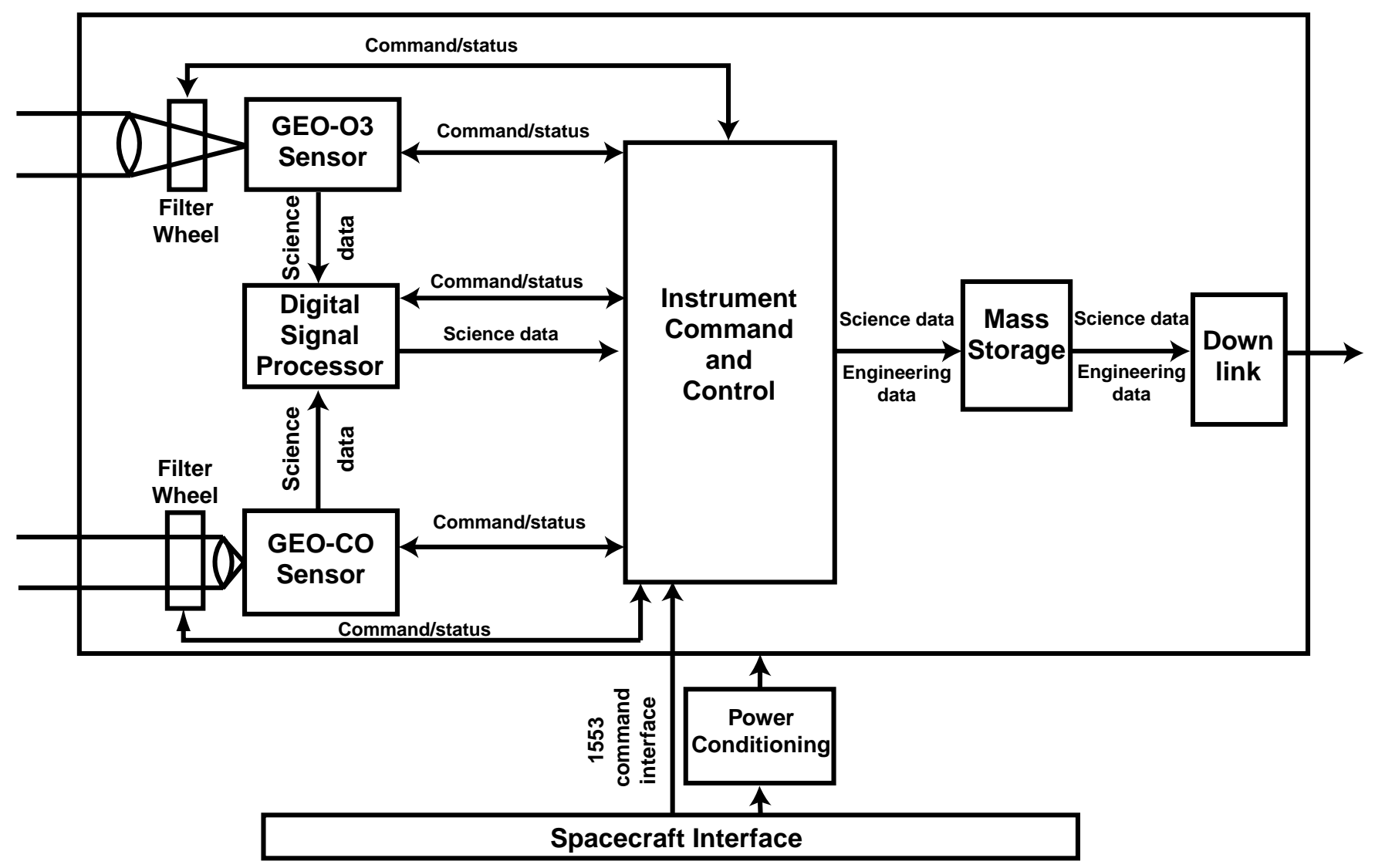

Figure 7 GEO TROPSAT block diagram.

\section{GEO-CO Sensor}

The GEO-CO ${ }^{5}$ sensor has one spectral band, $2.345 \mu \mathrm{m} \pm 0.035 \mu \mathrm{m}$, with three channels defined by the contents of the three gas cells. The GEO-CO sensor uses gas filter correlation radiometry (GFCR) to measure $\mathrm{CO}$, as well as a reference gas, $\mathrm{CH}_{4}$, in the near infrared portion of the spectrum. A filter wheel containing three gas cells, a cell with $\mathrm{CO}$, a cell with $\mathrm{CH}_{4}$, and a vacuum cell, is located in front of the optical system. The filter wheel spins continuously taking pictures through the cells as they pass in front of the optical system. The images are taken until the desired signal to noise ratio has been achieved in all three channels. A cooled interference filter, located directly in front of the focal plane array, is used to set the spectral band. The detector array is an advanced TE cooled hybrid $1024 \times 1024 \mathrm{HgCdTe}$ focal plane array with a $2.5 \mu \mathrm{m}$ cutoff wavelength. The CCD array is thermoelectrically cooled to $180 \mathrm{~K}$ to minimize the dark current.

GEO-TROPSAT detector parameters are summarized in Table 3. 


\begin{tabular}{||c|c|c|c|c|c|c||}
\hline \hline \multicolumn{7}{|c||}{ Summary of Detector Parameters } \\
\hline Sensor & Technology & Channels & Array Size & Field of View (deg) & IFOV (deg) & $\begin{array}{c}\text { Detector Pixel } \\
\text { Dimension }(\mu \mathrm{m})\end{array}$ \\
\hline GEO-O3 & Silicon CCD & 14 & $1024 \times 1024$ & 20 & 0.0195 & 12 \\
\hline GEO-CO & HgCdTe FPA & 3 & $1024 \times 1024$ & 20 & 0.0195 & 18.5 \\
\hline
\end{tabular}

Table 3 Summary of GEO TROPSAT detector parameters.

\section{SUMMARY}

This paper has presented the GEO TROPSAT mission concept, a proposed first mission of the next generation of scientific tropospheric remote sensing missions. The use of geostationary satellite platforms allows for previously unobtainable scientific measurements of highly variable tropospheric constituents. The geostationary vantage point allows for continuous observations over large portions of the Earth (approximately a third), while a constellation of geostationary instruments provides global coverage. The secondary payload approach is a viable method for significantly reducing the cost of scientific missions while simultaneously providing increased capability. Real public benefits result from fostering public understanding of atmospheric processes, enhancing science education, and delivering air quality data to the public via television. The GEO TROPSAT mission concept addresses key scientific issues in atmospheric chemistry, partners with industry as a secondary payload, and expands use of scientific data for the public good.

\section{ACKNOWLEDGEMENTS}

The authors would like to gratefully acknowledge the contributions by Richard Davis for the radiance calculations and modeling, by Scott Nolf, Kathy Stacy, Wayne Palmer, and Dee Bullock for their analysis and graphics work.

\section{REFERENCES}

1. G. Wetzel, H. Fischer, and H. Oelhaf, "Remote sensing of trace gases in the mid infrared spectral region from a nadir view", Applied Optics, Vol. 34, No. 3, pp. 467-479, 1995.

2. P.K. Bhartia, K.F. Klenk, D. Gordon, and A.J. Fleig, "Nimbus-7 Total Ozone Algorithm", Proc. $5^{\text {th }}$ Conference on Atmospheric Radiation American Meteorological Society, Baltimore, MD, 1983.

3. V.V. Rozanov, D. Diebel, R.J. Spurr, and J.P. Burrows, "GOMETRAN: A radiative transfer model for the satellite project GOME: the plane parallel version”, J. Geophys. Res., Vol. 120, No. D14, pp. 16683-16695, 1997.

4. N.S. Pougatchev and G.W. Sachse, "Characterization of Carbon Monoxide Measurements by Gas Filter Correlation Radiometers from Satellites", in Optical Remote Sensing of the Atmosphere, Vol. 5, OSA Technical Digest Series (Optical Society of America, Washington, D.C.), pp. 122-125, 1997.

5. G.W. Sachse, N. S. Pougatchev, R.V. Hess, A.D. Little, P.L. Rinsland, and D.O. Neil, "Geo-Stationary Imaging of CO and $\mathrm{CH}_{4}$ Distributions: Instrument Concept", in Optical Remote Sensing of the Atmosphere, Vol. 5, OSA Technical Digest Series (Optical Society of America, Washington, D.C.), pp. 149-151, 1997. 